Physics letters A, 347, N4-6, (2005), 157-159.

\title{
UNIQUENESS OF THE SOLUTION TO INVERSE OBSTACLE SCATTERING PROBLEM
}

\author{
A. G. RAMM \\ MATHEMATICS DEPARTMENT, KANSAS STATE UNIVERSITY, \\ MANHATTAN, KS 66506-2602, USA
}

\begin{abstract}
It is proved that the scattering amplitude known at a fixed frequency, a fixed direction of the incident plane wave and all directions of the scattered wave in a solid angle, however small, determine uniquely the shape of a strictly convex obstacle with a smooth but not analytic boundary on which the Dirichlet boundary condition is assumed.
\end{abstract}

\section{INTRODUCTION AND MAIN RESULT.}

Let $D \subset \mathbb{R}^{3}$ be a bounded domain with a smooth (say, twice continuously differentiable), but not analytic, boundary $S$,

$$
\begin{gathered}
\left(\nabla^{2}+k^{2}\right) u=0 \quad \text { in } \quad D^{\prime}:=\mathbb{R}^{3} \backslash D, \quad k=\text { const }>0 ; \quad u=0 \quad \text { on } \quad S, \\
u=\exp (i k \alpha \cdot x)+A\left(\alpha^{\prime}, \alpha, k\right) r^{-1} \exp (i k r)+o\left(r^{-1}\right), \quad r:=|x| \rightarrow \infty, \quad \alpha^{\prime}:=x r^{-1} .
\end{gathered}
$$

Here $\alpha \in S^{2}$ is a given unit vector, $S^{2}$ is the unit sphere in $\mathbb{R}^{3}$, the function $A\left(\alpha^{\prime}, \alpha, k\right)$ is called the scattering amplitude (the radiation pattern). It is known [1] that problem (1)-(2) has a unique solution, called the scattering solution. The inverse obstacle scattering problem (IP) is:

Given $A\left(\alpha^{\prime}, \alpha, k\right)$ on a subset of $S^{2} \times S^{2} \times[0, \infty)$ find $S$.

The first uniqueness result for IP was proved in 1964 by M.Schiffer. This result says:

The data $A\left(\alpha^{\prime}, \alpha_{0}, k\right)$, known for all $\alpha^{\prime} \in S^{2}$, a fixed $\alpha=\alpha_{0} \in S^{2}$, and all $k \in[0, \infty)$, determine $S$ uniquely.

In this result the Dirichlet boundary condition was assumed on the boundary. In [1], p.86, the result of M.Schiffer is proved and the Dirichlet boundary condition is not assumed a priori, but it is proved that the boundary condition is uniquely determined by the above scattering data.

A modification of Schiffer's argument, given by A.G.Ramm ([2], p.238), allows one to prove uniqueness theorem for the Neumann boundary condition. The original Schiffer's argument used the discreteness of the spectrum of the Dirichlet Laplacian in a bounded domain, but the Neumann Laplacian in some (nonsmooth) bounded domains may have continuous spectrum, and the modified argument in [2] has removed this difficulty.

The second result, proved in 1985 by A.G.Ramm, says:

The data $A\left(\alpha^{\prime}, \alpha, k_{0}\right)$, known at a fixed $k=k_{0}>0$ for all $\alpha \in S_{1}^{2}$ and all $\alpha^{\prime} \in S_{2}^{2}$, where $S_{j}^{2}, j=1,2$, are arbitrary small open subsets of $S^{2}$, (i.e. solid angles), determine $S$ and the boundary condition on $S$ uniquely.

This result is proved in [1], p.87.

The boundary conditions in Ramm's uniqueness theorem could be the Dirichlet, or the Neumann, or the Robin condition. A.G. Ramm has proved ([1], p.62) that $A\left(\alpha^{\prime}, \alpha, k\right)$ is analytic with respect to $\alpha$ and $\alpha^{\prime}$ on the variety $M:=\left\{z: z \cdot z=1, z \in \mathbb{C}^{3}\right\}$, where $z \cdot \zeta:=\sum_{j=1}^{3} z_{j} \zeta_{j}$, and meromorphic with respect to $k \in \mathbb{C}$. Thus, Schiffer's uniqueness theorem holds if the data $A\left(\alpha^{\prime}, \alpha_{0}, k\right)$ are known for all $k \in(a, b)$, where $0 \leq a<b$ are arbitrary fixed numbers, and all $\alpha^{\prime} \in S_{1}^{2}$.

For several decades the uniqueness theorem for IP with the non-overdetermined data $A\left(\alpha^{\prime}\right):=A\left(\alpha^{\prime}, \alpha_{0}, k_{0}\right)$, known for a fixed $k=k_{0}>0$, a fixed $\alpha=\alpha_{0} \in S^{2}$, and all $\alpha^{\prime} \in S_{1}^{2}$, has not been proved. An obvious

1991 Mathematics Subject Classification. 78A45, 35R30; PACS 0340Kf.

Key words and phrases. wave scattering, inverse obstacle scattering problem, non-overdetermined scattering data. 
consequence (mentioned in [2]) of the argument used in the proof of the uniqueness result in [1], p.85, is the following observation:

If the obstacle is known a priori to be so small that $k^{2}$ is below the lowest Dirichlet eigenvalue of the Laplace operator in $D$, then the above data determine $S$ uniquely.

It was also observed in [2] that the same argument proves the following claim:

The data $A\left(\alpha^{\prime}, \alpha_{j}, k_{0}\right), 1 \leq j \leq J$, known for sufficiently large $J$, determine $S$ uniquely.

Here $J$ should be so large that the total number of the Dirichlet eigenvalues of the Laplacian in $D$ below $k^{2}$ should be less than $J([2]$, p.237). In [2], p. 236-240, uniqueness theorems are obtained for inverse obstacle scattering for rough obstacles and for transmission boundary conditions, and the ideas, different from the original Schiffer's idea, were used.

Our aim in this paper is to prove a uniqueness theorem with the data $A\left(\alpha^{\prime}\right)$ assuming $S$ strictly convex, $C^{2}$-smooth but not an analytic set, i.e., not a set of zeros of an analytic function. In other words, the equation of the boundary is not of the form $f\left(x_{1}, x_{2}, x_{3}\right)=0$, where $f$ is an analytic function of its three variables in a neihgborhood of any point which belongs to the surface $S$ (see, e.g., [6] for a discussion of analytic sets and analytic surfaces).

Theorem 1. The data $A\left(\alpha^{\prime}\right)$ determine uniquely a strictly convex obstacle $D$ with $C^{2}-$ smooth boundary which is not an analytic set.

\section{PROOFS.}

By the remark, made in the Introduction, about analytic properties of the scattering amplitude, one may assume that $A\left(\alpha^{\prime}\right)$ is known for all $\alpha^{\prime} \in S^{2}$. Assume that $D_{1}$ and $D_{2}$ generate the same data $A\left(\alpha^{\prime}\right)$. Denote $D_{3}=D_{1} \cup D_{2}, D_{4}=D_{1} \cap D_{2}$. Let $S_{4}$ be the boundary of $D_{4}$. Since $D_{1}$ and $D_{2}$ are strictly convex, there are two cases to consider.

Case 1 is: $D_{4}$ does not contain interior points. Case 2 is: $D_{4}$ contains interior points. Let us show that both cases lead to a contradiction provided that $D_{1}$ and $D_{2}$ are strictly convex smooth bodies. If this is shown, then $D_{1}=D_{2}$, and Theorem 1 is proved.

Case 1. If $D_{4}$ does not contain interior points, then it is either empty or consists of one point. In both cases the argument is the same. In these cases $u_{1}=u_{2}$ in $D_{3}^{\prime}:=\mathbb{R}^{3} \backslash D_{3}$ by Lemma 1 in [1], p.25, where $u_{j}$ is the scattering solution for the obstacle $D_{j}$. Indeed, $u:=u_{1}-u_{2}$ solves equation (1) in $D_{3}^{\prime}$ and $u=o\left(\frac{1}{r}\right)$ as $r \rightarrow \infty$ because the scattering amplitudes are the same for $D_{1}$ and $D_{2}$ by our assumption. Thus $\lim _{r \rightarrow \infty} \int_{|s|=r}|u|^{2} d s=0$. This and the cited Lemma 1 imply $u=0$ in $D_{3}^{\prime}$. If $D_{4}$ is empty and $u_{1}=u_{2}$ in $D_{3}^{\prime}$, then $u_{1}$ is defined in $D_{2}$ and $u_{2}$ is defined in $D_{1}$, and $w$, which is equal to $u_{1}$ in $D_{1}^{\prime}$ and to $u_{2}$ in $D_{2}^{\prime}$, is defined in the whole space $\mathbb{R}^{3}$, solves there equation (1), and has asymptotics of the type (2). Therefore $v:=w-e^{i k \alpha \cdot x}$ solves equation (1) in $\mathbb{R}^{3}$ and satisfies the radiation condition. Consequently $v=0$ in $\mathbb{R}^{3}$ (see [1]), and $w=e^{i k \alpha \cdot x}$. This is a contradiction because $w=u_{1}=0$ on $S_{1}$. Thus, Case 1 cannot occur.

Case 2. If $D_{4}$ is not empty, and $D_{j}, j=1,2$, are strictly convex, then either $S_{1}$ intersects $S_{2}$ (call it case 2.1), and in this case there must be a point $P \in S_{4}$ at which the jump $\psi$ of the slope of the tangent line to $S_{4}$ is greater than $\pi$, or one of the bodies lies inside the other (call it case 2.2). Without loss of generality we may assume in this case that $D_{2} \subset D_{1}$.

In case 2.1 the solutions $u_{1}=u_{2}:=U$ in a neighborhood of $P$ must be of order $O\left(r^{\gamma}\right)$ as $r \rightarrow 0$, where $r$ is the distance between $x \in\left(D_{4}^{\prime} \cap D_{3}^{\prime}\right)$ and $P$, and $\gamma:=\frac{\psi}{\pi}, 1<\gamma<2$. This follows from the known results about behavior of solutions to Helmholtz and Laplace equations near a conical point of the boundary on which the Dirichlet boundary condition holds (see, e.g., [3], Chapter 1, Section 3, problems 3 and 4 , and [4]). Therefore $\nabla U$ is unbounded as $r \rightarrow 0$. This contradicts the fact that $u_{1}$ and $u_{2}$ are $C^{1}-\operatorname{smooth}$ up to the boundaries $S_{1}$ and $S_{2}$ respectively. The smoothness of the solution to the scattering problems up to the smooth boundary is a known fact (see, e.g., [5]).

In case 2.2 we may assume that $D_{2}$ is strictly inner subset of $D_{1}$ because otherwise $S_{1}$ and $S_{2}$ would have common arc and at the point, where this arc ends, the argument used in Case 2.1 would yield a contradiction. If $D_{2}$ is strictly inner subset of $D_{1}$, then $S_{1}$, the boundary of $D_{1}$, is an analytic set, because it is a set of zeros of the solution $u_{2}$ of equation (1), which is a real analytic function in $D_{2}^{\prime}$. Since we have assumed that the boundary is not an analytic set, the case 2.2 cannot occur. 
Theorem 1 is proved.

Remark. In the case 2.2 it is not possible to eliminate the possibility of the existence of $S_{2}$ such that $D_{2} \subset D_{1}$ and $u$ vanishes on $S_{1}$ and on $S_{2}$, and is of the form $u=u_{0}+v$, where the incident field $u_{0}$ solves equation (1), but is not necessarily a plane wave, and $v$ satisfies the usual radiation condition. For example, if $u_{0}=\frac{e^{-i k r}}{r}$, where $r=|x|, x \in \mathbb{R}^{3}$, and $v=b \frac{e^{i k r}}{r}$, where $b=e^{2 i \pi p}$, and $p$ is a real number, then $u=u_{0}+v$ solves equation (1) and $v$ satisfies the radiation condition. If $S_{r}$ is a ball of radius $r$, then $u=0$ on $S_{r}$ provided that $r=r_{n}:=\frac{(n-p) \pi}{k}$, where $n$ is an arbitrary integer. In particular, by choosing $p=1-\frac{k}{\pi}$ and $n=1$, one obtains $u=0$ on the sphere of radius 1 , and also on all the spheres of radii $r_{n}=\frac{n \pi}{k}-\left(1-\frac{k}{\pi}\right) \frac{\pi}{k}$, where $n$ is an arbitrary integer. This example does not exclude the possibility that for a special incident field, for example, for $u_{0}=e^{i k \alpha \cdot x}$ it is not possible to find $D_{2} \subset D_{1}$ such that $u=0$ on $S_{1}$ and on $S_{2}$, so that $u$ solves the scattering problem in $D_{1}^{\prime}$ and in $D_{2}^{\prime}$ and $D_{1} \neq D_{2}$.

\section{REFERENCES}

[1] A. G. Ramm, Scattering by Obstacles, Reidel, Dordrecht (1986).

[2] A.G. Ramm, Inverse Problems, Springer, New York (2005).

[3] L. Landau and E. Lifshitz, Electrodynamics of Continuous Medium, Pergamon Press, Oxford (1984).

[4] S. Nazarov and B. Plamenevsky, Elliptic Problems in Domains with Piecewise Smooth Boundaries, de Gruyter, New York (1994).

[5] D. Gilbarg and N. Trudinger, Elliptic Partial Differential Equations of Second Order, Springer-Verlag, New York (1998).

[6] B.Fuks, Theory of Analytic Functions of Several Complex Variables, Amer. Math. Soc., Providence (1963).

E-mail address: ramm@math.ksu.edu

Website: http://www.math.ksu.edu/ ramm 